\title{
Associated factors in distinguishing patients with brucellosis from suspected cases
}

Jingjing Luo, Huixin Yang, Fangfang Hu, Siwen Zhang, Taijun Wang, Qian Zhao, Ruize Wang and Qing Zhen*

\begin{abstract}
Background: To investigate the risk factors for brucellosis in suspected cases of the disease.

Methods: A self-designed questionnaire was developed to collect data from 3557 people whose initial visit site was the Songyuan Center for Disease Control and Prevention (CDC) from January 1st, 2009 to December 31st, 2012. After collecting blood samples, a plate agglutination test (PAT) and serum agglutination test (SAT) were used to distinguish the patients with brucellosis from the suspected cases.

Results: Sex, occupation (farmers and herdsmen), contact with abortion products, and contact with feces were the main risk factors for brucellosis in the suspected cases (all $P<0.05$ ). No difference existed between the confirmed cases and suspected cases in the demographic characteristics, contact with animals (except swine), contact with substances, or clinical symptoms (except fever). However, the confirmed cases showed significant differences from people without brucellosis in demographic characteristics, contact with animals (except cattle and swine), contact with substances, and clinical symptoms. Suspected cases exhibited significant differences from people without brucellosis in the demographic characteristics (except education), contact with animals (except swine), contact with substances (except dust), and clinical symptoms (except chills and acratia). Brucella was cultured from the blood samples of three of 30 suspected cases with fever. Using AMOS-PCR and agarose electrophoresis, the detailed species of Brucella strain was identified as Brucella melitensis.
\end{abstract}

Conclusions: Abortion products and feces are the main risk factors for brucellosis in suspected cases of the disease. Pyrexia in suspected cases with a history of contact with abortion products or feces should raise suspicion for the disease.

Keywords: Brucellosis, Suspected case, Confirmed case

\section{Background}

Brucellosis is the most common zoonosis caused by Brucella infection. The disease is classified as one of the category $\mathrm{B}$ infectious diseases in China. According to reports, the average annual growth rate of brucellosis in $2003-2014$ is $20.8 \%$, and it will continue to rise over the next 5 years [1].

The symptoms of human brucellosis include undulant fever, weight loss, night sweats, joint pain, enlarged lymph nodes and hepatosplenomegaly. Because the

\footnotetext{
* Correspondence: zhenqing@jlu.edu.cn

Department of Epidemiology and Biostatistics, Key Laboratory of Zoonosis Research, Ministry of Education, Jilin University School of Public Health, No.1163, Xinmin Street, Chaoyang District, Changchun, Jilin 130021, People's

Republic of China
}

clinical manifestations of brucellosis are diverse and nonspecific, a missed or incorrect diagnosis for brucellosis is possible, especially for clinically suspected cases [2-5]. Clinically suspected brucellosis cases are defined as individuals with clinical manifestations and epidemiological profiles who test positive by the plate agglutination test (PAT). In fact, clinically suspected brucellosis cases include individuals with suboptimal health, misdiagnosed brucellosis cases, and patients with other diseases [6]. The clinically suspected cases lack standardized treatment and management protocols. Some of these suspected cases may develop chronic brucellosis, which poses a serious burden for treatment [7].

(c) The Author(s). 2019 Open Access This article is distributed under the terms of the Creative Commons Attribution 4.0 International License (http://creativecommons.org/licenses/by/4.0/), which permits unrestricted use, distribution, and reproduction in any medium, provided you give appropriate credit to the original author(s) and the source, provide a link to the Creative Commons license, and indicate if changes were made. The Creative Commons Public Domain Dedication waiver (http://creativecommons.org/publicdomain/zero/1.0/) applies to the data made available in this article, unless otherwise stated. 
In this article, we investigated the risk factors of the confirmed cases, suspected cases, and people without brucellosis to raise awareness among physicians and suspected cases.

\section{Methods}

Definitions

The diagnosis of brucellosis was based on the "Diagnostic criteria for brucellosis" (WS269-2007).

\section{A confirmed case}

A confirmed case was defined (1) by epidemiological history; (2) by characteristic clinical findings and (3) as having either positive blood cultures for Brucella or a serum agglutination brucella antibody titer of $\geq 1: 100$.

\section{A suspected case}

A suspected case was defined (1) by epidemiological history; (2) by characteristic clinical findings and (3) as having a standard plate agglutination titer of $\geq 0.04$ and a serum agglutination brucella antibody titer of $\leq 1: 50$.

\section{An asymptomatic infection}

The difference between a confirmed case and a person with asymptomatic infection is that the latter was free of clinical symptoms and no organs were damaged.

Except for the suspected cases, confirmed cases and people with asymptomatic infection, the remainder of the visitors to the Songyuan CDC from 2009 to 2012 were negative for brucellosis.

\section{Study protocol}

A self-designed questionnaire was used to collect information, including demographic characteristics (sex, age, nation, education level, and occupation), contact with animals, manner of contact and clinical symptoms (Additional file 1), and the initial visit site was the Songyuan Center for Disease Control and Prevention (CDC) from January 1st, 2009 to December 31st, 2012. We excluded those cases that had a history of brucellosis and whose questionnaire missed important information that could not be supplemented, such as the exposure history and laboratory findings. Finally, we enrolled a total 3557 people (2860 with clinical symptoms and 697 without clinical symptoms).

Blood samples were collected from all the enrollees. Based on a titer of $<0.04$ detected by the plate agglutination test (PAT), we found 1939 people (1487 with clinical symptoms and 452 without clinical symptoms) without brucellosis. Based on the criteria of titer of $\geq 1$ : 100 or $\leq 1: 50$ with the serum agglutination test (SAT), we determined 991 confirmed symptomatic cases, 382 suspected cases, 169 confirmed asymptomatic cases, and 76 people without brucellosis. Because brucellosis is characterized by the acute or insidious onset of fever and one or more symptoms, including night sweats, arthralgia, headache, fatigue, anorexia, myalgia, weight loss, arthritis/spondylitis, meningitis, or focal organ involvement (endocarditis, orchitis/epididymitis, hepatomegaly, splenomegaly), we randomly chose 30 suspected cases with fever to investigate the possibility of diagnosing brucellosis using the Brucella culture and validation with agarose electrophoresis or AMOS-PCR products [8] (Fig. 1). The sequence of PCR primers is listed in Additional file 2.

\section{Data management and analysis}

Normally distributed data were displayed as the mean and standard deviation $(x \pm \mathrm{s})$. The median and Q1 to Q3 (25th to 75th percentiles, respectively) are shown. The chi-square test was used to compare the demographic characteristics, contact route, and clinical symptoms among the three groups (i.e., confirmed cases, suspected cases, and people without brucellosis). When the expected values in any of the cells of a contingency table were below 1 or more than $20 \%$ of the cells had an expected count less than 5, Fisher's exact test was used. The difference revealed by the chi-square test was further analyzed using the Bonferroni adjustment method. The adjusted significance level $\alpha$ was 0.017 , and statistical significance was attained when a $p$-value was less than this value. Multinomial logistic regression was used to confirm factors influencing the occurrence of brucellosis. The assignment of independent variables is shown in Table 1. For the brucellosis risk factors, we chose $P<$ 0.05 for the inclusion criteria, $0.05 \leq P \leq 0.10$ for the suspected risk criteria, and $P>0.1$ for the exclusion criteria. The data were calculated using Epi-Data version 3.1 software and STATA version 12.6.

\section{Results \\ Baseline characteristics}

From 2009 to 2012, a total 3557 individuals were enrolled in this study and further divided into three groups (confirmed cases: 991; suspected cases: 382; people without brucellosis: 2015). We compared the differences among the three groups using the chi-square test and found significant differences in demographic characteristics, contact history (except with deer, canine, dairy and meat), and clinical symptoms (except dizziness, cough, wrist pain, omalgia, sacroiliac pain, and lymphatic swelling) $(P<0.05)$ (Tables 2, 3 and 4).

We further investigated the difference between any two groups using the Bonferroni adjustment $(P<0.017)$. For the demographic characteristics, our results revealed no difference between the confirmed and suspected cases. However, the confirmed cases were significantly different from the people without brucellosis, and the 


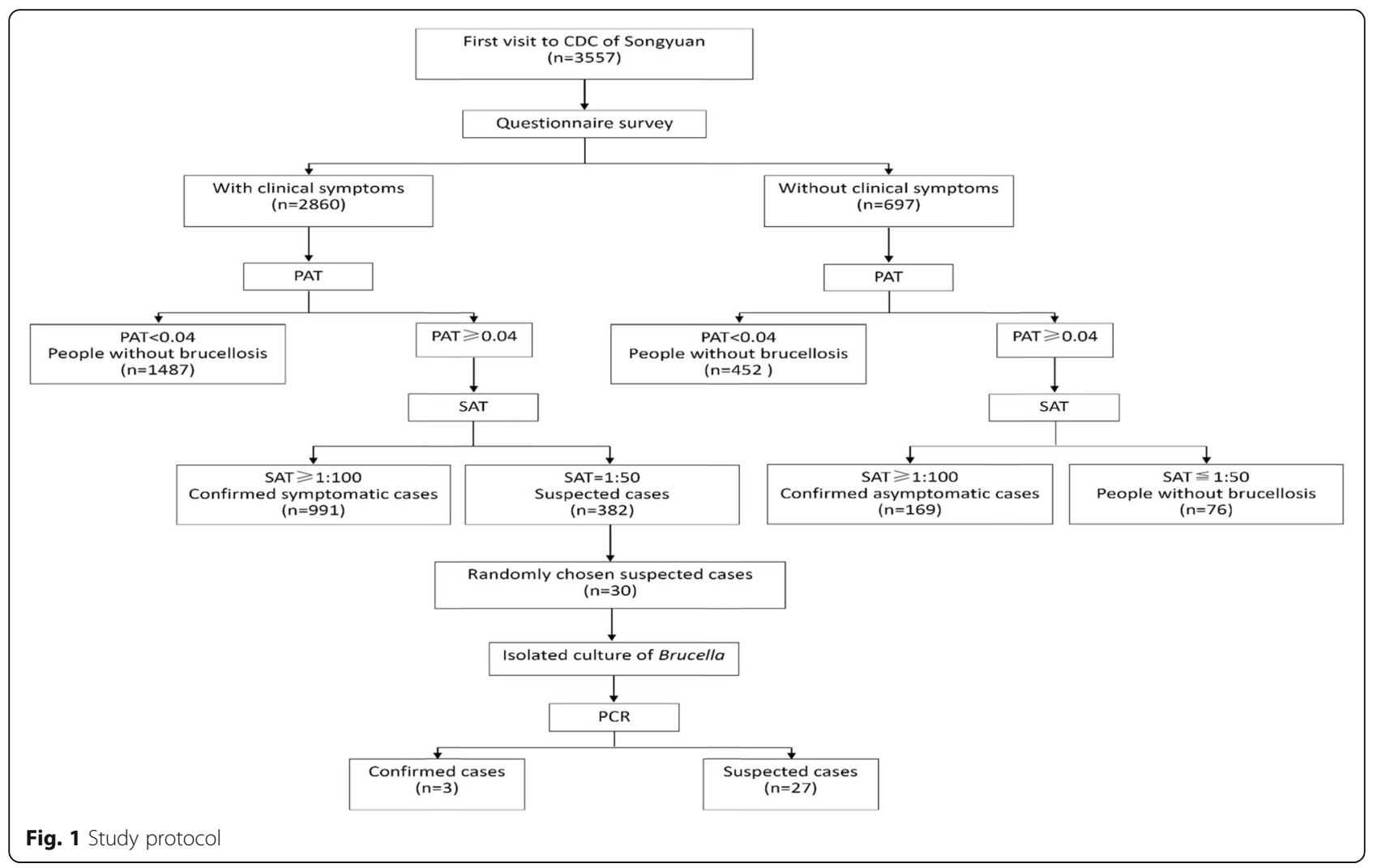

suspected cases exhibited significant differences from the people without brucellosis (except in education, $P=$ 0.557) (Table 2).

After comparing the history of contact with animals between the confirmed and suspected cases, we found that only contact with swine was a significant characteristic $(P=0.002)$. Nevertheless, contact with swine showed no difference between the confirmed cases and the people without brucellosis $(P=$ 0.012 ) or between the suspected cases and the people without brucellosis $(P=0.029)$. For contact with sheep, significant differences existed between the confirmed cases and the people without brucellosis and between the suspected cases and the people without brucellosis (all $P<0.001$ ). Moreover, for contact with cattle, the people without brucellosis showed no difference with the confirmed cases $(P=0.021)$ but exhibited a significant difference with the suspected cases $(P=0.002)$. In addition, with respect to contact with substances, our results indicated no difference between the confirmed and suspected cases, but both the confirmed and suspected cases exhibited significant differences with the people without brucellosis (except for contact

Table 1 Assignment of independent variables

\begin{tabular}{ll}
\hline Variable & Assignment \\
\hline Sex & Men $=1$, Women $=2$ \\
Age & $\begin{array}{l}66 \sim 86 \text { years old }=1,56 \sim 65 \text { years old }=2,46 \sim 55 \text { years old =3, 36 45 years old =4, 26 35 years old =5, 14 25 years old =6, } \\
1 \sim 13 \text { years old }=7\end{array}$ \\
Education & Primary $=1$, Junior $=2$, Senior $=3$, Undergraduate and above =4, Unknown =5, Illiteracy =6 \\
Occupation & Farmer and herdsmen =1, Unknown =2, Nonfarmer and nonherdsmen =3 \\
Contact with abortion & Yes $=1$, No $=2$ \\
Contact with fur & Yes $=1$, No $=2$ \\
Contact with feces & Yes $=1$, No $=2$ \\
Contact with dust & Yes $=1$, No $=2$ \\
Family member of & Yes $=1$, No $=2$ \\
infected & \\
\hline
\end{tabular}


Table 2 Demographic characteristics of the participants

\begin{tabular}{|c|c|c|c|c|c|c|c|c|c|c|c|c|c|c|c|c|c|c|}
\hline \multirow{3}{*}{ Sex } & \multirow{2}{*}{\multicolumn{2}{|c|}{$\begin{array}{l}\text { Confirmed } \\
\text { case } \\
n(\%)\end{array}$}} & \multirow{2}{*}{\multicolumn{2}{|c|}{$\begin{array}{l}\text { Suspected } \\
\text { case } \\
\text { n (\%) }\end{array}$}} & \multirow{2}{*}{\multicolumn{2}{|c|}{$\begin{array}{l}\text { People } \\
\text { without } \\
\text { brucellosis } \\
\mathrm{n}(\%)\end{array}$}} & \multicolumn{3}{|c|}{$\mathrm{C}$ vs S vs Pe } & \multicolumn{3}{|c|}{ C vs S } & \multicolumn{3}{|c|}{$\mathrm{C}$ vs Pe } & \multicolumn{3}{|c|}{$\mathrm{S}$ vs Pe } \\
\hline & & & & & & & \multicolumn{2}{|c|}{$x^{2}$} & \multirow{2}{*}{$\begin{array}{l}P \\
<0.001\end{array}$} & \multicolumn{2}{|c|}{$x^{2}$} & \multirow{2}{*}{$\begin{array}{l}P \\
0.049\end{array}$} & \multicolumn{2}{|c|}{$x^{2}$} & \multirow{2}{*}{$\begin{array}{l}P \\
<0.001\end{array}$} & \multicolumn{2}{|c|}{$x^{2}$} & $P$ \\
\hline & & & & & & & 89 & .141 & & 3 & .877 & & 82 & .664 & & 18 & .482 & $<0.001$ \\
\hline Men & 759 & (76.59) & 273 & (71.47) & 1205 & (59.80) & & & & & & & & & & & & \\
\hline Women & 232 & $(23.41)$ & 109 & (28.53) & 810 & $(40.20)$ & & & & & & & & & & & & \\
\hline Age & & & & & & & 64 & .861 & $<0.001$ & 10 & .32 & 0.112 & 36 & .423 & $<0.001$ & 34 & .573 & $<0.001$ \\
\hline$\leq 13$ & 21 & $(2.12)$ & 6 & $(1.57)$ & 132 & $(6.55)$ & & & & & & & & & & & & \\
\hline $14 \sim 25$ & 99 & (9.99) & 24 & $(6.28)$ & 234 & $(11.61)$ & & & & & & & & & & & & \\
\hline $26 \sim 35$ & 162 & $(16.35)$ & 69 & (18.06) & 364 & (18.06) & & & & & & & & & & & & \\
\hline $36 \sim 45$ & 281 & (28.36) & 128 & (33.51) & 524 & $(26.00)$ & & & & & & & & & & & & \\
\hline $46 \sim 55$ & 271 & $(27.35)$ & 92 & (24.08) & 470 & (23.33) & & & & & & & & & & & & \\
\hline $56 \sim 65$ & 129 & $(13.02)$ & 56 & (14.66) & 225 & (11.17) & & & & & & & & & & & & \\
\hline$\geq 66$ & 28 & (2.83) & 7 & (1.83) & 66 & (3.28) & & & & & & & & & & & & \\
\hline Education & & & & & & & 21 & .509 & 0.018 & 5 & .452 & 0.340 & 18 & .901 & 0.002 & 3 & .947 & 0.557 \\
\hline Illiteracy & 52 & $(5.25)$ & 15 & (3.93) & 116 & $(5.76)$ & & & & & & & & & & & & \\
\hline Primary edu & 611 & $(61.65)$ & 234 & $(61.26)$ & 1202 & (59.65) & & & & & & & & & & & & \\
\hline Junior edu & 296 & $(29.87)$ & 112 & (29.32) & 559 & $(27.74)$ & & & & & & & & & & & & \\
\hline Senior edu & 27 & (2.72) & 17 & $(4.45)$ & 99 & $(4.91)$ & & & & & & & & & & & & \\
\hline Undergraduate \& above & 3 & $(0.30)$ & 3 & $(0.79)$ & 29 & $(1.44)$ & & & & & & & & & & & & \\
\hline Unknown & 2 & $(0.20)$ & 1 & $(0.26)$ & 10 & $(0.50)$ & & & & & & & & & & & & \\
\hline Occupation & & & & & & & 155 & .747 & $<0.001$ & 12 & .754 & 0.415 & 123 & .857 & $<0.001$ & 54 & .654 & $<0.001$ \\
\hline Farmers and herdsmen & 838 & $(84.56)$ & 331 & $(86.65)$ & 1527 & (75.78) & & & & & & & & & & & & \\
\hline Livestock merchant & 6 & $(0.61)$ & 3 & $(0.79)$ & 21 & $(1.04)$ & & & & & & & & & & & & \\
\hline Livestock slaughterer & 9 & $(0.91)$ & 1 & $(0.26)$ & 14 & $(0.69)$ & & & & & & & & & & & & \\
\hline Dairy processor & 6 & $(0.61)$ & 2 & $(0.52)$ & 7 & $(0.35)$ & & & & & & & & & & & & \\
\hline Fur-making worker & 2 & $(0.20)$ & 0 & $(0.00)$ & 2 & $(0.10)$ & & & & & & & & & & & & \\
\hline Other worker & 10 & $(1.01)$ & 4 & $(1.05)$ & 60 & $(2.98)$ & & & & & & & & & & & & \\
\hline Veterinarian & 3 & $(0.30)$ & 2 & $(0.52)$ & 7 & $(0.35)$ & & & & & & & & & & & & \\
\hline Doctor or nurse & 0 & $(0.00)$ & 1 & $(0.26)$ & 6 & $(0.30)$ & & & & & & & & & & & & \\
\hline Student & 13 & $(1.31)$ & 7 & $(1.83)$ & 75 & $(3.72)$ & & & & & & & & & & & & \\
\hline Children & 12 & $(1.21)$ & 2 & $(0.52)$ & 79 & $(3.92)$ & & & & & & & & & & & & \\
\hline Unemployed & 3 & $(0.30)$ & 0 & $(0.00)$ & 35 & $(1.74)$ & & & & & & & & & & & & \\
\hline Officer & 0 & $(0.00)$ & 2 & $(0.52)$ & 30 & $(1.49)$ & & & & & & & & & & & & \\
\hline Freelancer & 25 & $(2.52)$ & 7 & $(1.83)$ & 99 & $(4.91)$ & & & & & & & & & & & & \\
\hline Unknown & 64 & $(6.46)$ & 20 & $(5.24)$ & 53 & $(2.63)$ & & & & & & & & & & & & \\
\hline
\end{tabular}

with dust in the suspected cases vs the people without brucellosis, $P=0.323$ ) (Table 3 ).

When comparing the clinical symptoms, we found no difference between the confirmed and suspected cases (except with fever, $P=0.004)$. However, the confirmed cases were significantly different from the people without brucellosis $(P=0.065)$, and the suspected cases exhibited significant differences from the people without brucellosis $(P=0.373)$ (except with chills and acratia) (Table 4).

\section{Analysis of risk factors of brucellosis}

We used multivariable logistic regression to identify the risk factors for brucellosis, and our results demonstrated that sex (adjusted odds ratio [aOR]: 2.249; 95\% confidence interval [CI]: 1.864-2.712), age (14 86 years old) (aOR: 2.186; 95\% CI: 1.037-4.608), occupation (farmers and herdsmen, and unspecified occupation) (aOR: 1.434; 95\% CI: $1.052-1.953$ and aOR: 5.071; 95\% CI: 3.091-8.319, respectively), and 
Table 3 Contact history of the participants

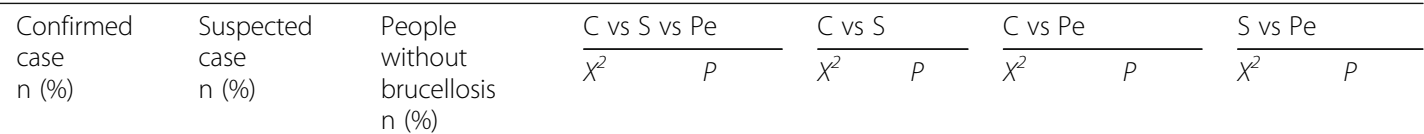

\begin{tabular}{|c|c|c|c|c|c|c|c|c|c|c|c|c|c|c|}
\hline \multicolumn{15}{|l|}{ Contact with animals } \\
\hline Cattle & & & & & & & 12.891 & 0.002 & 2.486 & 0.115 & 5.338 & 0.021 & 9.601 & 0.002 \\
\hline yes & 85 & (8.58) & 23 & $(6.02)$ & 228 & $(11.32)$ & & & & & & & & \\
\hline no & 906 & $(91.42)$ & 359 & (93.98) & 1787 & (88.68) & & & & & & & & \\
\hline Sheep & & & & & & & 65.415 & $<0.001$ & 1.217 & 0.270 & 58.904 & $<0.001$ & 17.383 & $<0.001$ \\
\hline yes & 654 & (65.99) & 240 & $(62.83)$ & 1032 & $(51.22)$ & & & & & & & & \\
\hline no & 337 & $(34.01)$ & 142 & $(37.17)$ & 983 & $(48.78)$ & & & & & & & & \\
\hline Swine & & & & & & & 9.649 & 0.008 & 9.742 & 0.002 & 2.532 & 0.112 & 4.785 & 0.029 \\
\hline yes & 64 & $(6.46)$ & 44 & $(11.52)$ & 163 & (8.09) & & & & & & & & \\
\hline no & 927 & (93.54) & 338 & $(88.48)$ & 1852 & (91.91) & & & & & & & & \\
\hline Deer & & & & & & & 0.582 & 0.843 & - & - & - & - & - & - \\
\hline yes & 5 & $(0.50)$ & 1 & $(0.26)$ & 7 & $(0.35)$ & & & & & & & & \\
\hline no & 986 & (99.50) & 381 & (99.74) & 2008 & $(99.65)$ & & & & & & & & \\
\hline Canine & & & & & & & 2.376 & 0.305 & - & - & - & - & - & - \\
\hline yes & 162 & $(16.35)$ & 59 & $(15.45)$ & 287 & $(14.24)$ & & & & & & & & \\
\hline no & 829 & $(83.65)$ & 323 & $(84.55)$ & 1728 & $(85.76)$ & & & & & & & & \\
\hline \multicolumn{15}{|c|}{ Contact with substances } \\
\hline Abortion products & & & & & & & 213.426 & $<0.001$ & 1.945 & 0.163 & 194.577 & $<0.001$ & 72.951 & $<0.001$ \\
\hline yes & 446 & $(45.01)$ & 156 & $(40.84)$ & 414 & $(20.55)$ & & & & & & & & \\
\hline no & 545 & $(54.99)$ & 226 & $(59.16)$ & 1601 & $(79.45)$ & & & & & & & & \\
\hline Dairy and meat & & & & & & & 4.182 & 0.124 & - & - & - & - & - & - \\
\hline yes & 34 & $(3.43)$ & 15 & (3.93) & 101 & $(5.01)$ & & & & & & & & \\
\hline no & 957 & $(96.57)$ & 367 & $(96.07)$ & 1914 & ((94.99) & & & & & & & & \\
\hline Fur & & & & & & & 43.090 & $<0.001$ & 0.011 & 0.915 & 35.375 & $<0.001$ & 16.015 & $<0.001$ \\
\hline yes & 649 & $(65.49)$ & 249 & $(65.18)$ & 1090 & (54.09) & & & & & & & & \\
\hline no & 342 & $(34.51)$ & 133 & (34.82) & 925 & (45.91) & & & & & & & & \\
\hline Feces & & & & & & & 84.272 & $<0.001$ & 0.370 & 0.543 & 66.699 & $<0.001$ & 45.770 & $<0.001$ \\
\hline yes & 231 & (23.31) & 95 & $(24.87)$ & 238 & (11.81) & & & & & & & & \\
\hline no & 760 & (76.69) & 287 & (75.13) & 1777 & (88.19) & & & & & & & & \\
\hline Dust & & & & & & & 15.424 & $<0.001$ & 2.490 & 0.115 & 15.426 & $<0.001$ & 0.977 & 0.323 \\
\hline yes & 305 & (30.78) & 101 & (26.44) & 485 & $(24.07)$ & & & & & & & & \\
\hline no & 686 & (69.22) & 281 & (73.56) & 1530 & (75.93) & & & & & & & & \\
\hline
\end{tabular}

contact with abortion products (aOR: 2.513; 95\% CI: 2.040-3.096) were significantly associated with the risk of brucellosis in the confirmed cases (all $P<$ 0.05 ). In addition, sex (aOR: 1.652; 95\% CI: $1.284-$ 2.126), occupation (farmers and herdsmen; unspecified occupation) (aOR: 1.895; 95\% CI: 1.195-3.004 and aOR: 4.646; 95\% CI: 2.341-9.220, respectively), contact with abortion products (aOR: 2.186; 95\% CI: 1.037-4.608), and contact with feces (aOR: 1.506; 95\% CI: 1.039-2.183) were significantly associated with the risk of brucellosis in the suspected cases (all $P<0.05$ ) (Table 5).

\section{Germ culture of blood samples}

To investigate the possibility of diagnosing brucellosis in the suspected cases, we randomly chose blood samples from 30 suspected cases with fever to culture Brucella and found the bacterium in the blood samples of three cases. Furthermore, we used AMOS-PCR and agarose electrophoresis to identify the detailed 
Table 4 Clinical symptoms of the participants

\begin{tabular}{|c|c|c|c|c|c|c|c|c|c|c|c|c|c|c|c|}
\hline & \multirow{2}{*}{\multicolumn{2}{|c|}{$\begin{array}{l}\text { Confirmed } \\
\text { case } \\
\text { n (\%) }\end{array}$}} & \multirow{2}{*}{\multicolumn{2}{|c|}{$\begin{array}{l}\text { Suspected } \\
\text { case } \\
\mathrm{n}(\%)\end{array}$}} & \multirow{2}{*}{\multicolumn{2}{|c|}{$\begin{array}{l}\text { People } \\
\text { without } \\
\text { brucellosis } \\
\mathrm{n}(\%)\end{array}$}} & \multicolumn{3}{|c|}{ C vs S vs Pe } & \multicolumn{2}{|c|}{ C vs S } & \multicolumn{2}{|l|}{ C vs Pe } & \multicolumn{2}{|l|}{$\mathrm{S}$ vs Pe } \\
\hline & & & & & & & $\overline{x^{2}}$ & & $P$ & $x^{2}$ & $P$ & $x^{2}$ & $P$ & $x^{2}$ & $P$ \\
\hline Fever & & & & & & & 330 & .505 & $<0.001$ & 8.466 & 0.004 & 300.494 & $<0.001$ & 86.074 & $<0.001$ \\
\hline yes & 732 & (73.86) & 252 & $(65.97)$ & 811 & $(40.25)$ & & & & & & & & & \\
\hline no & 259 & $(26.14)$ & 130 & (34.03) & 1204 & $(59.75)$ & & & & & & & & & \\
\hline Chills & & & & & & & 10 & .363 & 0.006 & 0.081 & 0.776 & 9.314 & 0.002 & 3.409 & 0.065 \\
\hline yes & 117 & $(11.81)$ & 43 & $(11.26)$ & 168 & $(8.34)$ & & & & & & & & & \\
\hline no & 874 & (88.19) & 339 & (88.74) & 1847 & (91.66) & & & & & & & & & \\
\hline Acratia & & & & & & & 7 & .303 & 0.026 & 0.812 & 0.367 & 7.235 & 0.007 & 0.794 & 0.373 \\
\hline yes & 392 & (39.56) & 141 & (36.91) & 696 & (34.54) & & & & & & & & & \\
\hline no & 599 & $(60.44)$ & 241 & (63.09) & 1319 & $(65.46)$ & & & & & & & & & \\
\hline Hyperhidrosis & & & & & & & 119 & .031 & $<0.001$ & 3.369 & 0.066 & 113.247 & $<0.001$ & 30.548 & $<0.001$ \\
\hline yes & 369 & (37.24) & 122 & (31.94) & 389 & $(19.31)$ & & & & & & & & & \\
\hline no & 622 & $(62.76)$ & 260 & $(68.06)$ & 1626 & (80.69) & & & & & & & & & \\
\hline Dizziness & & & & & & & 0 & .490 & 0.783 & - & - & - & - & - & - \\
\hline yes & 21 & $(2.12)$ & 8 & $(2.09)$ & 50 & $(2.48)$ & & & & & & & & & \\
\hline no & 970 & $(97.88)$ & 374 & $(97.91)$ & 1965 & $(97.52)$ & & & & & & & & & \\
\hline Headache & & & & & & & 57 & .392 & $<0.001$ & 0.222 & 0.638 & 51.125 & $<0.001$ & 21.371 & $<0.001$ \\
\hline yes & 235 & $(23.71)$ & 86 & $(22.51)$ & 269 & $(13.35)$ & & & & & & & & & \\
\hline no & 756 & (76.29) & 296 & (77.49) & 1746 & $(86.65)$ & & & & & & & & & \\
\hline Cough & & & & & & & 0 & .153 & 0.926 & - & - & - & - & - & - \\
\hline yes & 20 & $(2.02)$ & 8 & $(2.09)$ & 45 & $(2.23)$ & & & & & & & & & \\
\hline no & 971 & (97.98) & 374 & $(97.91)$ & 1970 & $(97.77)$ & & & & & & & & & \\
\hline Joint and muscle pain & & & & & & & 63 & .716 & $<0.001$ & 0.158 & 0.691 & 55.357 & $<0.001$ & 23.535 & $<0.001$ \\
\hline yes & 359 & (36.23) & 134 & (35.08) & 470 & $(23.33)$ & & & & & & & & & \\
\hline no & 632 & $(63.77)$ & 248 & $(64.92)$ & 1545 & $(76.67)$ & & & & & & & & & \\
\hline Muscular soreness & & & & & & & 24 & .294 & $<0.001$ & 0.387 & 0.534 & 17.532 & $<0.001$ & 14.175 & $<0.001$ \\
\hline yes & 150 & (15.14) & 63 & (16.49) & 200 & $(9.93)$ & & & & & & & & & \\
\hline no & 841 & $(84.86)$ & 319 & $(83.51)$ & 1815 & $(90.07)$ & & & & & & & & & \\
\hline Omalgia & & & & & & & 2 & .676 & 0.262 & - & - & - & - & - & - \\
\hline yes & 43 & $(4.34)$ & 16 & (4.19) & 65 & (3.23) & & & & & & & & & \\
\hline no & 948 & (95.66) & 366 & (95.81) & 1950 & $(96.77)$ & & & & & & & & & \\
\hline Wrist pain & & & & & & & 0 & .582 & 0.837 & - & - & - & - & - & - \\
\hline yes & 5 & $(0.50)$ & 1 & $(0.26)$ & 7 & $(0.35)$ & & & & & & & & & \\
\hline no & 986 & (99.50) & 381 & (99.74) & 2008 & (99.65) & & & & & & & & & \\
\hline Lumbago & & & & & & & 41 & .410 & $<0.001$ & 2.899 & 0.089 & 41.003 & $<0.001$ & 6.558 & 0.010 \\
\hline yes & 198 & (19.98) & 61 & (15.97) & 228 & $(11.32)$ & & & & & & & & & \\
\hline no & 793 & $(80.02)$ & 321 & (84.03) & 1787 & (88.68) & & & & & & & & & \\
\hline Coxalgia & & & & & & & 19 & .362 & $<0.001$ & 0.092 & 0.762 & 15.361 & $<0.001$ & 10.847 & 0.001 \\
\hline yes & 63 & $(6.36)$ & 26 & $(6.81)$ & 66 & $(3.28)$ & & & & & & & & & \\
\hline no & 928 & (93.64) & 356 & (93.19) & 1949 & $(96.72)$ & & & & & & & & & \\
\hline Sacroiliac pain & & & & & & & 0 & .925 & 0.738 & - & - & - & - & - & - \\
\hline yes & 3 & $(0.30)$ & 2 & $(0.52)$ & 6 & $(0.30)$ & & & & & & & & & \\
\hline no & 988 & (99.70) & 380 & (99.48) & 2009 & (99.70) & & & & & & & & & \\
\hline
\end{tabular}


Table 4 Clinical symptoms of the participants (Continued)

\begin{tabular}{|c|c|c|c|c|c|c|c|c|c|c|c|c|c|c|c|}
\hline \multirow{3}{*}{ Gonalgia } & \multirow{2}{*}{\multicolumn{2}{|c|}{$\begin{array}{l}\text { Confirmed } \\
\text { case } \\
\mathrm{n}(\%)\end{array}$}} & \multirow{2}{*}{\multicolumn{2}{|c|}{$\begin{array}{l}\text { Suspected } \\
\text { case } \\
\text { n (\%) }\end{array}$}} & \multirow{2}{*}{\multicolumn{2}{|c|}{$\begin{array}{l}\text { People } \\
\text { without } \\
\text { brucellosis } \\
\text { n (\%) }\end{array}$}} & \multicolumn{3}{|c|}{ C vs S vs Pe } & \multicolumn{2}{|l|}{ C vs S } & \multicolumn{2}{|l|}{ C vs Pe } & \multicolumn{2}{|l|}{$\mathrm{S}$ vs $\mathrm{Pe}$} \\
\hline & & & & & & & \multicolumn{2}{|c|}{$x^{2}$} & \multirow{2}{*}{$\begin{array}{l}P \\
0.005\end{array}$} & \multirow{2}{*}{$\begin{array}{l}x^{2} \\
0.748\end{array}$} & \multirow{2}{*}{$\begin{array}{l}P \\
0.387\end{array}$} & \multirow{2}{*}{$\begin{array}{l}x^{2} \\
6.065\end{array}$} & \multirow{2}{*}{$\begin{array}{l}P \\
0.014\end{array}$} & \multirow{2}{*}{$\begin{array}{l}x^{2} \\
7.560\end{array}$} & \multirow{2}{*}{$\begin{array}{l}P \\
0.006\end{array}$} \\
\hline & & & & & & & 10 & .661 & & & & & & & \\
\hline yes & 140 & (14.13) & 61 & (15.97) & 222 & $(11.02)$ & & & & & & & & & \\
\hline no & 851 & (85.87) & 321 & (84.03) & 1793 & (88.98) & & & & & & & & & \\
\hline Dolor vagus & & & & & & & 27 & .616 & $<0.001$ & 0.143 & 0.705 & 21.253 & $<0.001$ & 13.960 & $<0.001$ \\
\hline yes & 224 & (22.60) & 90 & (23.56) & 317 & (15.73) & & & & & & & & & \\
\hline no & 767 & (77.40) & 292 & (76.44) & 1698 & $(84.27)$ & & & & & & & & & \\
\hline Lymphatic swelling & & & & & & & 4 & .580 & 0.079 & - & - & - & - & - & - \\
\hline yes & 2 & $(0.20)$ & 3 & $(0.79)$ & 3 & $(0.15)$ & & & & & & & & & \\
\hline no & 989 & (99.80) & 379 & $(99.21)$ & 2012 & (99.85) & & & & & & & & & \\
\hline Enlargement of testis & & & & & & & 21 & .422 & $<0.001$ & 0.232 & 0.630 & 20.433 & $<0.001$ & 7.883 & 0.005 \\
\hline yes & 42 & $(4.24)$ & 14 & (3.66) & 31 & (1.54) & & & & & & & & & \\
\hline no & 949 & (95.76) & 368 & (96.34) & 1984 & $(98.46)$ & & & & & & & & & \\
\hline
\end{tabular}

C Confirmed case; S Suspected case; Pe People without brucellosis

Primary edu: primary education; Junior edu: Junior middle school education; Senior edu: Senior middle school education

species of the Brucella strains and found the species to be Brucella melitensis (Fig. 2).

\section{Discussion}

Brucellosis is a common zoonotic infection caused by Brucella. This bacterial disease has not only a considerable influence on human and animal health but also a major socioeconomic impact because of loss in husbandry $[9,10]$. Each year, there are $5,000,000$ to 6,000 ,
000 brucellosis patients and 500,000 new cases worldwide [11]. Furthermore, most cases of brucellosis are underdiagnosed and underreported because of vague flulike symptoms, nonstandard medications, and difficulty in diagnosis [12].

Routine laboratory tests for the diagnosis of brucellosis include the PAT and SAT. If PAT and SAT identify suspected cases and trigger physicians to pay close attention to persistent symptoms in these suspected cases, Brucella

Table 5 Influencing factors of brucellosis by multinomial logistic regression. Reference: people without brucellosis; sex of control: women; age of control: 1 to 13 years old; profession of control: nonfarmer and nonherdsmen; contact history of control: no contact

\begin{tabular}{|c|c|c|c|c|c|c|c|c|c|c|c|c|c|c|c|}
\hline \multirow{3}{*}{$\begin{array}{l}\text { Group } \\
\text { Confirmed case }\end{array}$} & \multirow{3}{*}{$\begin{array}{l}\text { Influencing factor } \\
\text { Sex }\end{array}$} & \multirow{3}{*}{0} & \multirow[b]{3}{*}{.810} & \multirow{2}{*}{\multicolumn{2}{|c|}{ Std. Error }} & \multicolumn{2}{|c|}{ Wald } & \multirow{2}{*}{\multicolumn{2}{|c|}{ Sig. }} & \multirow{2}{*}{\multicolumn{2}{|c|}{$\operatorname{Exp}(B)$}} & \multicolumn{4}{|c|}{$95 \% \mathrm{Cl}$ for $\operatorname{Exp}(\mathrm{B})$} \\
\hline & & & & & & & & & & & & \multicolumn{2}{|c|}{ Lower } & \multicolumn{2}{|c|}{ Upper } \\
\hline & & & & 0 & .096 & 71 & .773 & 0 & .000 & 2 & .249 & 1 & .864 & 2 & .712 \\
\hline & \multicolumn{15}{|l|}{ Age } \\
\hline & 14 25-years-old & 0 & .652 & 0 & .327 & 3 & .966 & 0 & .046 & 1 & .920 & 1 & .010 & 3 & .647 \\
\hline & 26 35-years-old & 0 & .674 & 0 & .323 & 4 & .351 & 0 & .037 & 1 & .963 & 1 & .042 & 3 & .700 \\
\hline & 36 45-years-old & 0 & .790 & 0 & .316 & 6 & .269 & 0 & .012 & 2 & .204 & 1 & .187 & 4 & .091 \\
\hline & 46 55-years-old & 0 & .889 & 0 & .315 & 7 & .966 & 0 & .005 & 2 & .432 & 1 & .312 & 4 & .507 \\
\hline & 56 65-years-old & 0 & .917 & 0 & .325 & 7 & .947 & 0 & .005 & 2 & .501 & 1 & .322 & 4 & .730 \\
\hline & 66 86-years-old & 0 & .782 & 0 & .381 & 4 & .224 & 0 & .040 & 2 & .186 & 1 & .037 & 4 & .608 \\
\hline & Farmers and herdsmen & 0 & .360 & 0 & .158 & 5 & .218 & 0 & .022 & 1 & .434 & 1 & .052 & 1 & .953 \\
\hline & Unspecified occupation & 1 & .624 & 0 & .253 & 41 & .333 & 0 & .000 & 5 & .071 & 3 & .091 & 8 & .319 \\
\hline & Abortion products & 0 & .922 & 0 & .106 & 75 & .005 & 0 & .000 & 2 & .513 & 2 & .040 & 3 & .096 \\
\hline \multirow[t]{5}{*}{ Suspected case } & Sex & 0 & .502 & 0 & .129 & 15 & .240 & 0 & .000 & 1 & .652 & 1 & .284 & 2 & .126 \\
\hline & Farmers and herdsmen & 0 & .639 & 0 & .235 & 7 & .388 & 0 & .007 & 1 & .895 & 1 & .195 & 3 & .004 \\
\hline & Unspecified occupation & 1 & .536 & 0 & .350 & 19 & .288 & 0 & .000 & 4 & .646 & 2 & .341 & 9 & .220 \\
\hline & Abortion products & 0 & .714 & 0 & .144 & 24 & .629 & 0 & .000 & 2 & .043 & 1 & .541 & 2 & .708 \\
\hline & Feces & 0 & .409 & 0 & .189 & 4 & .670 & 0 & .031 & 1 & .506 & 1 & .039 & 2 & .183 \\
\hline
\end{tabular}




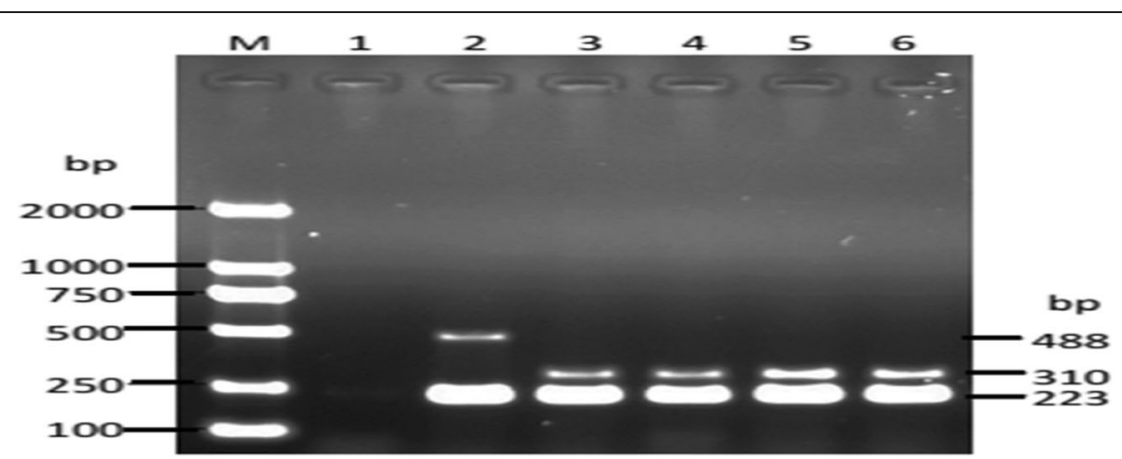

Fig. 2 Agarose electrophoresis of AMOS-PCR products. PCR amplification using B4/B5, AMOS-M-F, AMOS-A-F, AMOS-R primers identify 3 brucella strains. M: DNA markers; 1: Negative control; 2: 544A; 3: 16 M; 4-6: Isolated strains

culture from blood samples is a further determining method. Using the Brucella culture, Basappa G. Mantur found that $7.14 \%$ of suspected cases had brucellosis with negative PAT and SAT results [6]. Over 30\% of clinically suspected cases are confirmed by this method to have brucellosis [13]. Similarly, we used this method and found that $10 \%$ of clinically suspected cases had brucellosis. However, this method is still deficient in providing information on the Brucella strains $[14,15]$. Thus, we used AMOS-PCR to further determine the Brucella strains. Interestingly, the only strain we identified was Brucella melitensis. Because sheep-raising is the main economic source for farmers and herdsmen in Songyuan, sheep are one of the animals with which they most frequently come into contact. Moreover, Brucella melitensis (sheep) has much higher pathogenicity for humans than Brucella abortus (cattle) or Brucella suis (pig) [16]. These reasons at least partly support our findings. For these reasons, Brucella melitensis is the most prevalent Brucella species in Songyuan, Jilin province [17].

A high incidence of brucellosis exists in this province. As an agro-pastoral region, sheep, cattle, swine, deer, and canine are the main livestock. There is no clear border between the feeding areas and living areas. Moreover, the livestock waste is not subjected to sanitary treatment [18]. Contact with livestock and substances are the main activities contributing to brucellosis in Songyuan. We investigated the contribution of contact with livestock (sheep, cattle, swine, deer, and canine) and contact with substances (abortion products, dairy and meat, fur, feces, and dust) to the brucellosis risk. Our results revealed that abortion products were a risk factor for brucellosis both in the confirmed and suspected cases. Notably, feces were a risk factor for brucellosis only in the suspected cases. This is probably because the excreta eliminated by livestock such as cattle and sheep into the litter or into the air is not treated in time, and the Brucella in the feces enters the air to form infectious aerosol particles which infect humans through the respiratory system. Studies have shown that dust in sand and air may carry Brucella and can be transmitted by inhalation of infectious aerosol particles [19]. Unlike contact with abortion products, contact with feces is an indirect method for the confirmed and suspected cases, and infection of the respiratory tract by Brucella is the main route of fecal transmission. However, we could not provide the follow-up findings and repetition results of serology in the suspected cases with respiratory findings. Additionally, physicians pay more attention to suspected cases and possibly inquire further about contact information from these cases. These reasons may provide an explanation for feces being a risk factor for brucellosis in the suspected cases.

During the epidemiological investigation in Songyuan, we found that sex, farmers, and herdsmen were also risk factors for brucellosis in both the confirmed and suspected cases. Interestingly, constituent ratios exhibited an increasing tendency from the confirmed cases to the suspected cases to the people without brucellosis, in student, officers and people with senior middle school education or undergraduate and above education (Table 2).

After comparing the clinical symptoms, we found that the constituent ratio of the pyrexia cases exhibited a decreasing tendency from the confirmed cases to the suspected cases to the people without brucellosis (Table 4). Because the constituent ratio of pyrexia in the suspected cases was significantly higher than that in the people without brucellosis $(P<0.001)$, physicians should pay much more attention to pyrexia in suspected cases.

The authors, Basappa G. Mantur [6], and Wand Yi [13] performed retrospective studies and found cases misdiagnosed with brucellosis. Intriguingly, Catherine Kansiime [20] performed a prospective study and found that $31.8 \%$ of suspected cases ultimately develop brucellosis. These results further confirm that suspected cases remain at risk of brucellosis.

This study has limitations. We found that age (14-86) was a risk factor for brucellosis in the confirmed cases 
but not in the suspected cases. We cannot provide a sufficient explanation for this discrepancy, which merits further study in the future.

\section{Conclusion}

Abortion products and feces are the main risk factors for brucellosis in confirmed and suspected cases, and feces was a risk factor for brucellosis only in the suspected cases. This study confirms the need for policy makers to educate farmers about health care, avoiding unprotected contact with animal abortion products or feces, and wearing masks as often as possible. In addition, pyrexia in suspected cases with a history of contact with abortion products and feces should raise suspicion for the disease. The authors suggest further investigation of the main route of fecal transmission in suspected cases.

\section{Supplementary information}

Supplementary information accompanies this paper at https://doi.org/10. 1186/s12879-019-4662-3.

Additional file 1. Case questionnaire - for outpatient of brucellosis.

Additional file 2. Oligonucleotide sequences of primers used in AMOSPCR.

\section{Abbreviations}

CDC: Center for Disease Control and Prevention; PAT: Plate agglutination test; SAT: Serum agglutination test

\section{Acknowledgements}

The authors express their gratitude to Plague and Brucellosis Prevention and Control Base, Chinese Centers for Disease Control and Prevention, Baicheng, Jilin, P.R.China for the support in publication of this article. Authors are thankful to Prof. Yi Cheng (Jilin University) for his guiding of this article.

\section{Authors' contributions}

LJ, YHX, HFF, ZQ conceived the study and involved in data collection. ZSW, WTJ and WRZ involved in laboratory analysis and critically reviewed the manuscript. LJJ wrote the first draft of the manuscript. All authors reviewed and approved the final version of the manuscript.

\section{Funding}

This study was supported by a competitive grant from the Science and Technology Department of Jilin Province, China (Project Number: $20191102007 Y Y$ ). The funding body had no role in database design, analysis, or writing the manuscript.

\section{Availability of data and materials}

The datasets used and/or analyzed during the current study are available from the corresponding author on reasonable request.

\section{Ethics approval and consent to participate}

The study was approved by Songyuan CDC. Informed consents were obtained from all the participants. For minors, parents or legal guardians provided consent on their behalf. Participants that tested positive for Brucella had received medical treatment from the CDC. All subjects gave signed, informed consent to participate in the study, which was approved by the Institutional Review Board (IRB), School of Public Health, Jilin University.

\section{Consent for publication}

Not applicable in this section.

\section{Competing interests}

The authors declare that they have no competing interests
Received: 13 March 2019 Accepted: 25 November 2019

Published online: 09 December 2019

\section{References}

1. Lai S, Zhou H, Xiong W, Gilbert M, Huang Z, Yu J, Yin W, Wang L, Chen Q, Li Y, et al. Changing epidemiology of human brucellosis, China, 1955-2014. Emerg Infect Dis. 2017;23(2):184-94.

2. Asiimwe BB, Kansiime C, Rwego IB. Risk factors for human brucellosis in agro-pastoralist communities of south western Uganda: a case-control study. Bmc Research Notes. 2015;8(1):1-6.

3. Keyoumu A, Tai XP, Aishan M. Study on Diagnosis and Treatment of 5 Misdiagnosed Cases of Acute Brucellosis. In: Practical Preventive Medicine; 2013.

4. Ducrotoy MJ, Ammary K, Lbacha HA, Zouagui Z, Mick V, Prevost L, Bryssinckx W, Welburn SC, Benkirane A. Narrative overview of animal and human brucellosis in Morocco: intensification of livestock production as a driver for emergence? Infect Dis Pov. 2015;4(1):1-21.

5. Chen Q, Lai S, Yin W, Zhou H, Li Y, Mu D, Li Z, Yu H, Yang W. Epidemic characteristics, high-risk townships and space-time clusters of human brucellosis in Shanxi Province of China, 2005-2014. BMC Infect Dis. 2016;16(1):760.

6. Mantur BG, Amarnath SK, Patil GA, Desai AS. Clinical utility of a quantitative rose Bengal slide agglutination test in the diagnosis of human brucellosis in an endemic region. Clin Lab. 2014;60(4):533-41.

7. Young EJ. Serologic diagnosis of human brucellosis: analysis of 214 cases by agglutination tests and review of the literature. Rev Infect Dis. 1991;13(3): 359-72.

8. Pathak AD, Dubal ZB, Karunakaran M, Doijad SP, Raorane AV, Dhuri RB, Bale MA, Chakurkar EB, Kalorey DR, Kurkure NV, et al. Apparent seroprevalence, isolation and identification of risk factors for brucellosis among dairy cattle in Goa, India. Comp Immunol Microbiol Infect Dis. 2016;47:1-6.

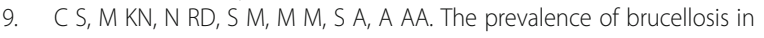
different provinces of Iran during 2013-2015. Iran J Public Health. 2019;48(1): 132-8.

10. Franc KA, Krecek RC, Hasler BN, Arenas-Gamboa AM. Brucellosis remains a neglected disease in the developing world: a call for interdisciplinary action. BMC Public Health. 2018;18(1):125.

11. Pappas G, Papadimitriou P, Akritidis N, Christou L, Tsianos EV. The new global map of human brucellosis. Lancet Infect Dis. 2006;6(2):91-9.

12. Godfroid J, Cloeckaert A, Liautard JP, Kohler S, Fretin D, Walravens K, Garinbastuji B, Letesson JJ. From the discovery of the Malta fever's agent to the discovery of a marine mammal reservoir, brucellosis has continuously been a re-emerging zoonosis. Vet Res. 2005;36(3):313.

13. Wang Y, Gao JW, Luo XD, Hui-Xia XI. Results of serological monitoring of human brucellosis from 2009 to 2013 in Ruzhou city. China Trop Med. 2015; 15(1):109-11.

14. Yunusa K. A review on diagnostic techniques for brucellosis. Afr J Biotechnol. 2014;13(1):1-10.

15. Du QC, Wang P. Advances in detection techniques of brucellosis. Chin J Animal Infect Dis. 2018;09(35):1-6.

16. Cao X, Li S, Li Z, Liu Z, Ma J, Lou Z, Zhou J, Liu Y, Jing Z, Fu B. Enzootic situation and molecular epidemiology of Brucella in livestock from 2011 to 2015 in Qingyang, China. Emerg Microbes Infect. 2018;7(1):58.

17. Zhen Q, Lu Y, Yuan X, Qiu Y, Xu J, Li W, Ke Y, Yu Y, Huang L, Wang Y. Asymptomatic brucellosis infection in humans: implications for diagnosis and prevention. Clin Microbiol Infect. 2013;19(9):E395-7.

18. Guan CL, Yuan ZC, Yao Y, Wang P, Liu FY, Wang RZ, Wang D, Li Y, Zhen Q. Suryey on awareness rate of brucellosis prevention knowledge and behavior prevalence among primary and secondary school students in breeding livestock's families in Western pastoral areas of Jilin province. J Jilin Univ (Medicine Edition). 2014;40(6):1303-7.

19. Griffin DW. Atmospheric movement of microorganisms in clouds of desert dust and implications for human health. Clin Microbiol Rev. 2007;20(3):45977 table of contents.

20. Kansiime C, Rutebemberwa E, Asiimwe BB, Makumbi F, Bazira J, Mugisha A. Annual trends of human brucellosis in pastoralist communities of southwestern Uganda: a retrospective ten-year study. Infect Dis Pov,4,1(2015-0831). 2015;4(1):39

\section{Publisher's Note}

Springer Nature remains neutral with regard to jurisdictional claims in published maps and institutional affiliations. 